\title{
CORRELATION BETWEEN WDF, WNR, AND RET ABNORMAL SCATTERGRAM DETECTED BY SYSMEX XN-1000 AND PARASITEMIA OF MALARIA PATIENTS IN MERAUKE HOSPITAL
}

\author{
Merylin Oktavia Ronoko ${ }^{1}$, Aryati $^{2}$, Arifoel Hajat ${ }^{2}$ \\ Laboratory Installation of Merauke Hospital, Merauke, Indonesia. E-mail: merylinronoko@yahoo.com \\ Department of Clinical Pathology, Faculty of Medicine, Airlangga University/Dr. Soetomo Hospital, Surabaya, Indonesia. E-mail: \\ dr_aryati@yahoo.com
}

\begin{abstract}
Malaria remains a health problem in Indonesia. Microscopic examination with Giemsa staining is the gold standard for diagnosing malaria. The density of parasites correlates with the degree of severity and response to therapy of malaria. Malaria-causing plasmodium can be detected by Sysmex XN-1000 which is marked by abnormalities in the WDF, WNR and RET scattergram. This research aimed to determine the correlation of WDF, WNR and RET abnormal scattergram detected by Sysmex XN-1000 and the parasitemia index of malaria at the Merauke General Hospital. This was a cross-sectional study with observational approach conducted between November 2017 - February 2018 at the Merauke General Hospital. Positive malaria samples were stained with Giemsa, their parasitemia index was calculated, routine complete blood count using Sysmex XN-1000 was performed, and the scattergram abnormalities were then analyzed. There were 65 positive malaria samples as follows: P.falciparum (35\%), P.vivax (60\%), P.ovale (3.1\%), and P.malariae (1.5\%), but the species did not correlate with parasitemic index $(p=0.691)$. Abnormalities of WDF and WNR scattergram were predominantly found than RET scattergram (80\% vs. 27.7\%). P.vivax predominantly caused abnormalities of the WDF and WNR scattergram in 36 of 39 samples (92.3\%), whereas P.falciparum predominantly caused abnomalities of the RET scattergram in 14 of 23 samples (60.9\%). There was 95\% positivity of an abnormality in WDF/WNR/RET scattergram with a cut-off of > 5,0165.5/ $\mu \mathrm{L}$. There was correlation between WDF, WNR, RET scattergram detected by Sysmex XN-1000 and the parasitemia index.
\end{abstract}

Key words: Malaria, scattergram abnormality, Sysmex XN-1000, parasitemia index

\section{INTRODUCTION}

Malaria remains a major global health problem, especially in tropical areas. The WHO predicted that in 2015 there were 214 million of new malaria cases, with 438 thousand deaths in the whole world, and one-third or 306 thousand cases of them were in infancy. ${ }^{1}$ In 2010 , there were 465,764 positive malaria cases in Indonesia, and there was a decrease as much as 209,413 cases in 2015. The National Annual Parasites Index (API) trend in 2011-2015 decreased from $1.75 \%$ (2011) to $0.85 \%$ (2015), but malaria cases were still high in endemic places, such as Papua, West Papua, Maluku, North Maluku, and Nusa Tenggara Timur. ${ }^{2}$

The Province of Papua had the highest malaria burden in Indonesia with API $45.85 \%$ in 2106 . All parasites of the Plasmodium species could be found in Papua, with P.falciparum causing the highest morbidity and mortality. ${ }^{3.4}$ The National Annual Parasites Index of the Regency of Merauke decreased from 19/1000 people in 2010 into
$15.28 / 1,000$ people in 2016, although malaria was still a health problem in this district.

Microscopic examination of malaria with Giemsa staining is still the gold standard for malaria examination. Microscopic examination can be used not only for diagnosis but also to evaluate therapy. The liability of this method is the subjectivity of the technicians, especially in diagnosing mixed infections or automated examination with a low parasite count. Detecting Plasmodium with an automated hematology analyzer is a new way to diagnose a patient with fever and suspected of malaria. Sysmex XN-1000, not only able to perform a complete blood count, it can also detect the presence of malaria-causing Plasmodium by an abnormality of the WDF, WNR, and RET scattergram. ${ }^{6}$

\section{METHODS}

This was an observational cross-sectional research. The inclusion criteria for this research were patient with positive laboratory results for malaria 
and who were willing to participate in this research by signing an informed consent. Patients who met the inclusion criteria were sent by clinicians to undergo another malaria examination. This study involved malaria patients from the emergency room, Specialist Inpatient Clinics and In-patients of Merauke General Hospital from November 2017 until February 2018.

The blood sampling and blood smears of patients infected by malaria were performed in the laboratory of Merauke General Hospital. Evaluation of the blood smear and parasite counting were carried out by two laboratory technicians with the supervision of a Clinical Pathologist. Discrepancies between the evaluation of the technicians were read by a third person. A total of 65 samples with were positive blood smear evaluation were determined the complete blood count using the Sysmex XN-1000. The abnormalities of the Sysmex XN-1000 scattergram were stated as positive and negative.

Statistical analysis used SPSS version 16.0. Data were analyzed and presented as tables, diagrams, and graphs. Malaria parasitic index in one of abnormalities of scattergrams (WDF/WNR/RET) was stated as positive or negative and analyzed using Mann Whitney test, with $\mathrm{p}<0.05$.

\section{RESULTS AND DISCUSSION}

The ethical committee of the Medical Faculty of Airlangga University Surabaya had approved and stated that this experiment was ethical with number
No. 37/EC/KEPK/FKUA/2018. The demographic characteristic of 65 subjects with malaria were shown in Table 1 . The subjects were dominantly males (48/65, $73.8 \%)$ with P.vivax as the main cause of the infection (39/60.0\%).

The ring, trophozoite and gametocyte stage of malaria-causing Plasmodium were shown in Figure 1.

The calculation of parasitemia index was reviewed by more than two experienced laboratory tehnicians. The mean of parasitemia index, the lowest parasitemia index, and the highest parasitemia index

Table 1. Demographic characteristics of malaria

\begin{tabular}{lc}
\hline Subjects Characteristics & Number \\
\hline Sex (\%) & $17(26.2 \%)$ \\
Female & $48(73.8 \%)$ \\
Male & \\
Age (years) & 29.86 \\
Mean & 13.807 \\
SD & 2 \\
Minimum & 64 \\
Maximum & \\
Type of malaria infection* & $25(35.4 \%)$ \\
P.falciparum (malaria tropicana) & $39(60.0 \%)$ \\
P.vivax (malaria tersiana) & $2(3.1 \%)$ \\
P.ovale (malaria ovale) & $1(1.5 \%)$ \\
P.malariae (malaria malariae) & \\
\hline * Type of malaria was determined based on microscopic \\
examination by blood smear evaluation and thick smear \\
stained by Giemsa
\end{tabular}
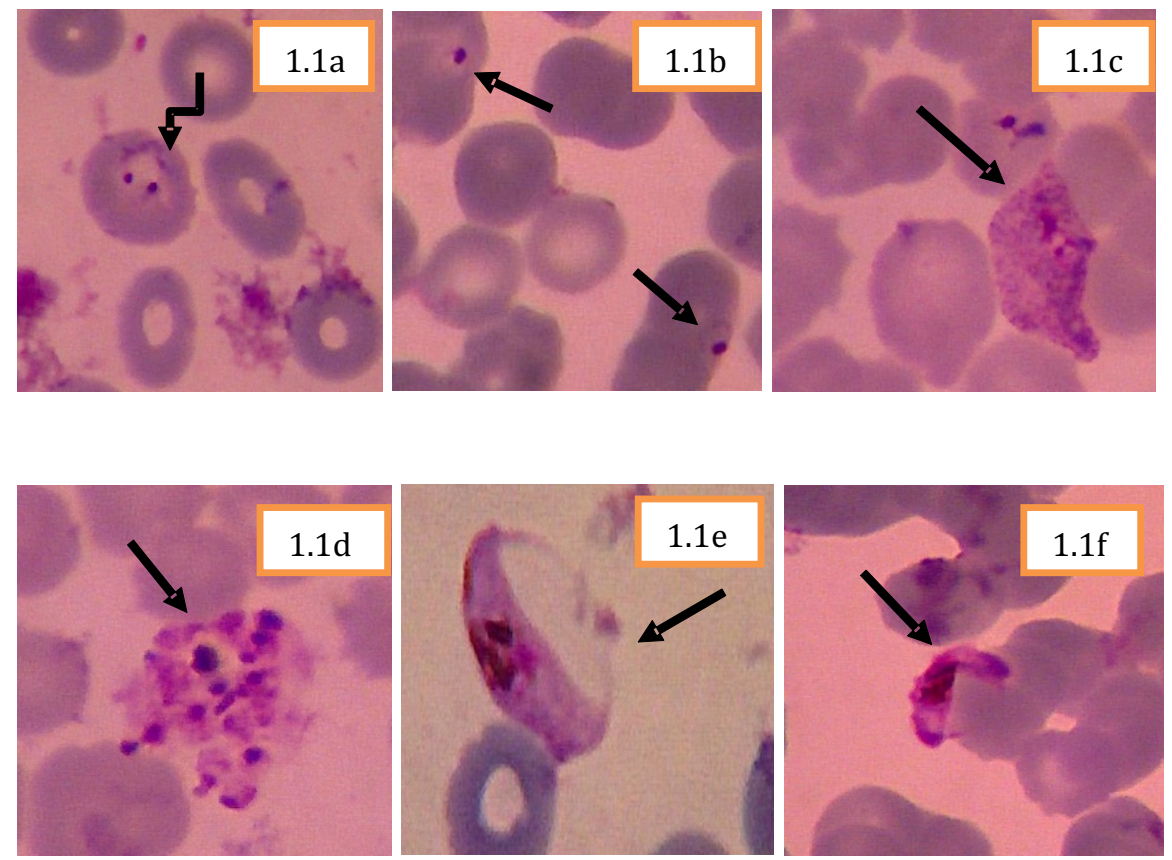

Figure 1. Plasmodium ring, trophozoite, schizont, and gametocyte stages shown by the arrowhead. (1.1a) Pv Ring; (1.1b) Pf Ring; (1.1c) Pv Trophozoite; (1.1d) Pf Mature Schizont; (1.1e) Pf male gametocyte; (1.1f) Pf female gametocyte 
was $11,312 \pm 18,682 / \mu \mathrm{L}, 67 / \mu \mathrm{L}$, and $143,100 / \mu \mathrm{L}$, respectively. The distribution of the parasitemia index of all three Plasmodium species was found in the range of $5,001-50,000 / \mu \mathrm{L}$.

\section{WDF and WNR scattergram}

This research showed that 9 of 65 infected subjects had no abnormalities in any scattergram channel. There were $80 \%$ infected samples that had abnormality of WDF/WNR channel. Abnormalities of WDF and WNR scattergram showed that Plasmodium falciparum had the lowest proportion compared to the other Plasmodium (Figure 2).

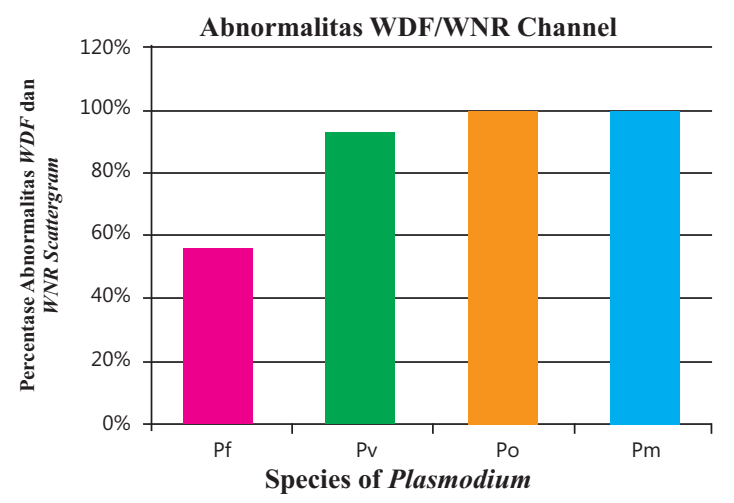

Figure 2. Percentage of abnormalities in WDF and WNR channels of Plasmodium-infected blood

Statistical analysis using Mann-Whitney test showed that there was no meaningful difference between parasitemia index with or without abnormalities of WDF and WNR scattergram $(p=0.241)$. This result showed that there was no correlation between parasitemia index with or without WDF and WNR scattergram abnormalities.

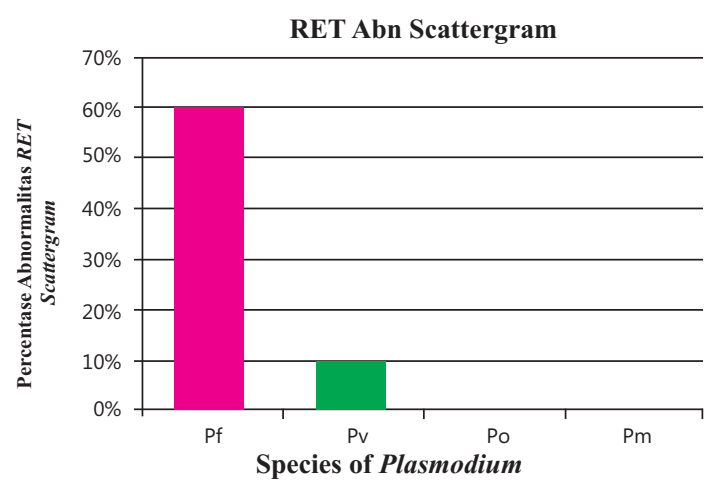

Figure 3. The percentage of abnormalities in WDF and WNR channel of Plasmodium-infected blood

\section{RET Scattergram}

This research showed abnormalities of RET scattergram in $27.7 \%$ of 65 samples, which was lower than abnormalities of WDF and WNR scattergram. Abnormalities in RET scattergram were $60.9 \%$ caused by P.falciparum (14/23 samples), whereas P.vivax only caused $10.3 \%$ (4/39) abnormalities (Figure 3).

This research did not show a significant difference between parasitemia index with or without abnormalities in the RET scattergram (Mann-Whitney test, $\mathrm{p}=0.317$ ). This showed that there was no correlation between malaria parasitemia index with or without abnormalities of the RET scattergram.

\section{WDF/WNR/RET Scattergram}

This research showed that there was one of scattergram abnormalities of WDF/WNR/RET in 56 samples infected with malaria-causing Plasmodium. Mann-Whitney test showed a significant difference of parasitemia index with or without abnormalities in one of scattergram (WDF/WNR/RET) $(p=0.022)$. This indicated that there was a significant correlation between one of scattergram abnormality (WDF/WNR/RET) with parasitemia index.

Statistical analysis using Receiver Operating Characteristic (ROC) curve to find the cut-off of parasitemia index to show an abnormality of WDF or WNR and/or RET scattergram could be seen in Figure 4.

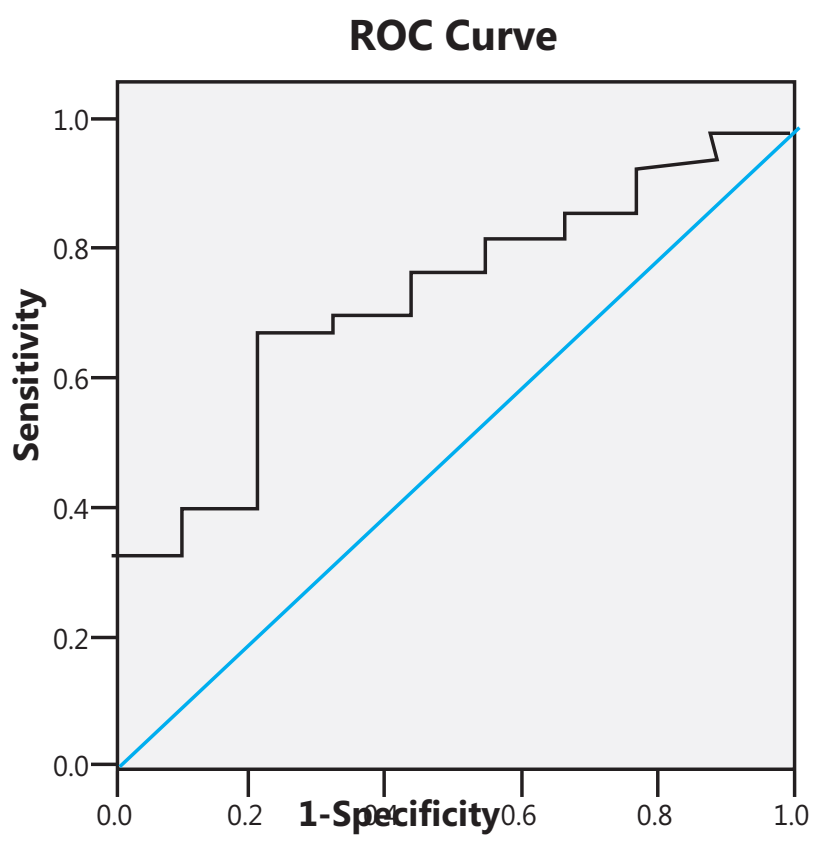

Figure 4. ROC curve analysis (AUC .739) for parasitemia index with a scattergram abnormality 
ROC curve analysis showed a cut-off $\geq 5,0165.5 / \mu \mathrm{L}$ (AUC $=0.739, \mathrm{Sn}=69.6 \%, \mathrm{Sp}=77.8 \%$ ). Parasitemia index $\geq 5,016.5 / \mu \mathrm{L}$ were found in 39 subjects $(95 \%)$ with abnormalities in one of the WDF/WNR/RET scattergram. There were 17 subjects (70.8\%) with a parasitemia index $<5,0165.5 / \mu \mathrm{L}$ and abnormalities in one of the WDF/WNR/RET scattergram. Statistical analysis showed that there was a suitability between parasitemia index with or without abnormalities in one of the WDF/WNR/RET scattergram (kappa test $=0.279, p=0.006$ ), with a moderate correlation between them.

Malaria targets all age groups in both males and females. Basic Health Research data in 2013 found the highest prevalence of malaria in the age group of 25 - 34 years old. Table 1 showed the higher proportion of males $(73.8 \%)$ infected with malaria compared to females (26.2\%). The age range of malaria patients was $2-64$ years old with a mean of $29.86 \pm 13.807$.

The accuracy of calculating the parasitemia index varied according to methods used. Several factors were able to cause mistakes in calculating parasitemia index. A way to lessen the probability of mistakes is using the number of leukocytes from the automated hematology analyzer as a reference. ${ }^{8}$ In this research, the parasitemia index used the leukocyte count from the Sysmex XN-1000.

The use of Sysmex XN-1000 based on the flow cytometry method shows three kinds of optical data about cells, such as the Forward Scatter Light (FSL) that shows cell size, Side Scatter (SSC) that shows the complexity of the cellular internal structures such as granules, and Side Fluorescence Light (SFL) that shows the contents of the cell nucleus. Hemozoin appears in a brown crystalline color produced by free heme released from hemoglobin catabolism and detoxification by malaria parasite. Hemozoine is phagocytized by neutrophils and monocytes. These cells can help identification of malaria-causing Plasmodium by automated methods that causes abnormalities of the WDF, WNR or RET channel. ${ }^{6}$

\section{WDF and WNR scattergram}

WDF scattergram will show abnormal cell clusters if the blood sample contains schizont and/or gametocytes. WDF scattergram will highly vary according to the parasite species. The mature an parasite and hemozoine crystal are more likely to be found in a blood sample infected by P.vivax and P.ovale compared to P.falciparum, leading to frequent WDF scattergram abnormalities caused by these two species. Blood samples containing gametocytes and/or schizonts can cause blue "ghost" cluster abnormalities of the WNR channel; therefore, use of the WNR scattergram is more accurate. ${ }^{9,10}$ This research was consistent with study Dumas et al. and Sysmex suggesting that the WDF scattergram were able to detect mature stages (schizont, gametocytes) of all Plasmodium species (except P.falciparum) in $80 \%$ cases of malaria. ${ }^{9}$

Other studies about WBC scattergram abnormalities have been reported. Sharma et al. suggested similar result as this study, that there was no correlation between the parasitemia index and WBC scattergram abnormality. ${ }^{10}$ Huh et al. and Yoo et al. stated that patients with pseudoeosinophilia were significantly correlated with the parasitemia index $(p<0.001) .{ }^{11}$

\section{RET Scattergram}

Plasmodium falciparum was more often observed in the RET scattergram, whereas P.vivax and P.ovale was rarely found in the RET scattergram. This was possibly caused by the ability of P.falciparum to infect all the stages of the erythrocyte (reticulocyte and mature erythrocyte), while the other Plasmodium species only infect reticulocytes or immature erythrocytes. Mature erythrocytes that are infected by P.falciparum resemble the reticulocyte (pseudoreticulocyte), which is detected by Sysmex XN-1000 as a reticulocyte and included in the RET calculation. ${ }^{12,13}$

Blood samples containing P.falciparum caused pseudoreticulocytes followed by an increase of reticulocyte count, without a change of the Immature Reticulocyte Fraction (IFR). The increase of LFR less than $90 \%$ and a RET Abn scattergram flagging would appear on the RET scattergram. This was not found in blood infected with P.vivax and P.ovale. ${ }^{12-14}$ Figure 3 showed that P.falciparum was the most dominant species (60.9\%) causing RET scattergram abnormalities which was in accordance with the study by Symex.

\section{WDF/WNR/RET Scattergram}

Positive values showed 95\% abnormalities in either WDF/WNR or RET scattergram with a parasitemia index of $\geq 5,016.5 / \mu \mathrm{L}$. This research showed that there was a correlation between abnormalities of the scattergram and malaria parasitemia index shown in one of the three scattergram channels. The higher the malaria parasitemia index, the more abnormality shown in one of the three channels (WDF/WNR/RET scattergram) and the more severe the infection. The 
limitation of this research was no description of clinical condition of the patient, so the correlation between the parasitemia index and severity of malaria was undetermined.

\section{CONCLUSION AND SUGGESTIONS}

There was a correlation between one of abnormality of WDF, WNR or RET scattergram detected by Sysmex XN-1000 and the parasitemia index of malaria patients in the Merauke Hospital. Another studies about the abnormalities of Sysmex $\mathrm{XN}$-1-scattergram in non-malaria patients and healthy subjects should be carried out.

\section{REFERENCES}

1. WHO malaria terminology. Available at http://www.who.int/malaria/publications/atoz/malari aterminology/en. Accessed August 31, 2017.

2. Kementerian Kesehatan Republik Indonesia. Malaria. Jakarta, Pusdatin Kemenkes. 2016.

3. Kementerian Kesehatan Republik Indonesia. Tular Vektor \& Zoonotik. Subdit Malaria - Ditjen P2P. 2017.

4. Permenkes RINo. 5, 2013.

5. Badan Pusat Statistik Merauke, 2014.

6. Sunilkumar KB, Naik P. Usefulness of automated hematology analyzer Sysmex XN 1000 in detection of Malaria. October-December 2016. Indian Journal of Pathology and Oncology, 2017; 3(4): 658-661. Available at http://oaji.net/articles/2017/17701483682902.pdf. Accessed August 31, 2017.

7. Riskesda 2013. Kemenkes. 2013. Accssed April 10, 2018. www.depkes.go.id/resources/download/ general/Hasil\%20Riskesdas\%202013.pdf.
8. Alves-Junior ER, Gomes LT, Assis-Oliveira FB, Silverio-Silva LR, Nery AF, et al. Quantification of parasite density in 2000 microscopic fields underestimates the parasitemia level in malaria parasites. Tropical Biomedicine, 2014; 31(2): 387-391. Available at: https://www.ncbi.nlm.nih.gov/ pubmed/25134910. Accessed April 10, 2018.

9. Dumas C, Bienvenu AL, Girard S, Picot S, Debize G, Durand B. Automated Plasmodium detection by Sysmex XN hematology analyzer. J Clin Pathol. 2018. Available at: https://www.ncbi.nlm.nih.gov/ pubmed/ 2929881. Accessed April 10, 2018.

10. Sharma S, Sethi N, Pujani M, Kushwaha S, Sehgal S. Abnormal WBC scattergram: A clue to the diagnosis of malaria. 2011. Available at: https://www.ncbi.nlm. nih.gov/pubmed/22980408. Accessed April 10, 2017.

11. Yoo JH, Song J, Lee-AK, Sun KY, Kim AY, et al. Automated detection of malaria-associated. 2010. Available at: https://www.ncbi.nlm.nih.gov/ pubmed/20207865. Accessed April 10, 2018.

12. Buero S, Manenti B, Seghezzi M, Moioli V, Bagorria M, Callegaro A, Ottomano C3, Lippi G. Abnormal scattergrams and cell population data generated by fully automated hematological analyzers: New tools for screening malaria infection?. 2017. Available at: https://www.ncbi.nlm.nih.gov/pubmed/29464900. Intl J Lab Hem. Accessed April 10, 2018.

13. Seed Haematology. Sysmex educational enhancement and development. 2015; 1-7.

14. Dubreuil $P$, Pihet $M$, Cau $S$, Croquefer $S$, Deguigne PA, et al. Use of Sysmex XE - 2100 and XE - 5000 hematology analyzers for the diagnosis of malaria in a nonendemic country (France). Intl Jnl Lab. Hem. 2013. Available at: https://onlinelibrary.wiley.com/ doi/pdf/10.1111/ijlh.12145. Accessed April 10, 2018. 